\title{
APORTACIÓN AL ESTUDIO SOBRE LA FENOLOGÍA REPRODUCTORA DE LAS ALGAS PARDAS Y VERDES DEL LITORAL DEL SURESTE DE ESPAÑA
}

\author{
Juan SOTO
}

\begin{abstract}
RESUMEN. En este trabajo se aporta información sobre la fenología reproductura de 30 táxones de algas pardas y 20 de algas verdes en el Sureste de España, comparando el comportamiento fenológico observado con el descrito en áreas adyacentes del mediterráneo español. Paralelamente, se interpreta la fenología detectada en base a las observaciones realizadas en el litoral y a la bibliografía consultada.
\end{abstract}

Palabras clave. Fenología reproductora, algas verdes, algas pardas, sureste de España.

SUMMARY. We provide information about the reproductive phenology of 30 taxa of brown algae and 20 of green ones in the South-East of Spain, comparing the phenologic behaviour observed with the one already described in adjoining areas of the Spanish Mediterranean. We also interpreted the reproductive phenology according to the observations made on the coast and the consulted bibliography.

Key words. Reproductive phenology, brown algae, green algae, suth-east of Spain.

\section{INTRODUCCIÓN}

Continuando los estudios sobre fenología reproductora de la algas marinas en el Sureste de España (Soto \& Conde, 1989) se hace en este trabajo una aportación a la fenología reproductora y de los talos de algas pardas y verdes.

Entre los factores reguladores de la fenología de macroalgas, Maggs \& Guiry (1987) citan temperatura, fotoperíodo, longitud de onda e irradiancia como los más importantes. Henry (1988) establece la luz y la temperatura como los factores más obvios, entre los ambientales, que regulan la reproducción de las algas pardas. Chapman \& Craige (1977) consideran que los niveles de nutrientes en el agua están implicados en la fenología de grandes feofíceas perennes, en base a estudios con Laminaria longicruris. En igual sentido incide Epiard-Lahaye (1988) en relación con 
Cystoseira stricta. Epiard-Lahaye et al. (1987) establecen también la influencia de los ritmos de emersión-inmersión sobre el crecimiento algal en base a estudios con el taxon anterior.

Como corolario se puede afirmar que las algas experimentan unos ritmos reproductores y de crecimiento que dependerán de su ubicación geográfica, ya que ésta, a su vez, condicionará los factores físico-químicos citados con anterioridad.

En el Mediterráneo español, secularmente, los estudios de fenología de campo de algas han sido parte de estudios más amplios acometidos a nivel regional. Aportan, generalmente, una información segmentaria debido al escaso período temporal en que se ha seguido la fenología de los táxones. Esto es especialmente significativo en especies de talo microscópico que pasan frecuentemente desapercibidas.

En el presente trabajo se aportará información sobre la fenología reproductura o de los talos de 30 táxones de algas pardas y 20 de algas verdes, comparándose con los datos existentes en otras áreas.

\section{MATERIAL Y MÉTODOS}

En el período de tiempo comprendido entre los años 1983 y 1989 se han realizado en el Sureste de España (entre los cabos de Palos y de Gata) 110 inventarios de bentos marino durante todos los meses del año. Para cada taxon de los existentes en los inventarios se ha determinado su estado reproductor en base al análisis de un número no inferior a cinco talos. Así se ha podido dar información, para cada taxon, a nivel organizativo de población.

Se ha establecido, subjetivamente, un número de observaciones igual o superior a diez para establecer un patrón fenológico suficientemente aproximado de la especie en esta región. Para los táxones menos frecuentemente observados no se da información en este trabajo.

Posteriormente se ha comparado el patrón fenológico en el Sureste de España con el existente en los litorales de las Islas Baleares (Gómez-Garreta, 1983), Valencia (Barceló, 1987), Mar Menor (excluído en nuestras observaciones, Pérez-Ruzafa, 1989) y Málaga (Conde, 1983). Ocasionalmente se hará referencia al litoral de Cádiz (Seoane, 1965) y al catalán (Polo, 1978) para las especies comunmente estudiadas.

\section{RESULTADOS}

Los resultados, para los táxones estudiados, se pueden apreciar en las tablas I y II. Para cada taxon, $\mathrm{N}$ indica el número de poblaciones estudiadas, $\mathrm{P}$ es el período de tiempo (meses) en que está presente y, según los órdenes, aparecen los períodos mensuales en que están presentes las diferentes estructuras reproductoras observadas: esporangios pluriloculares y uniloculares, esporocistos, gametociștos y receptáculos 


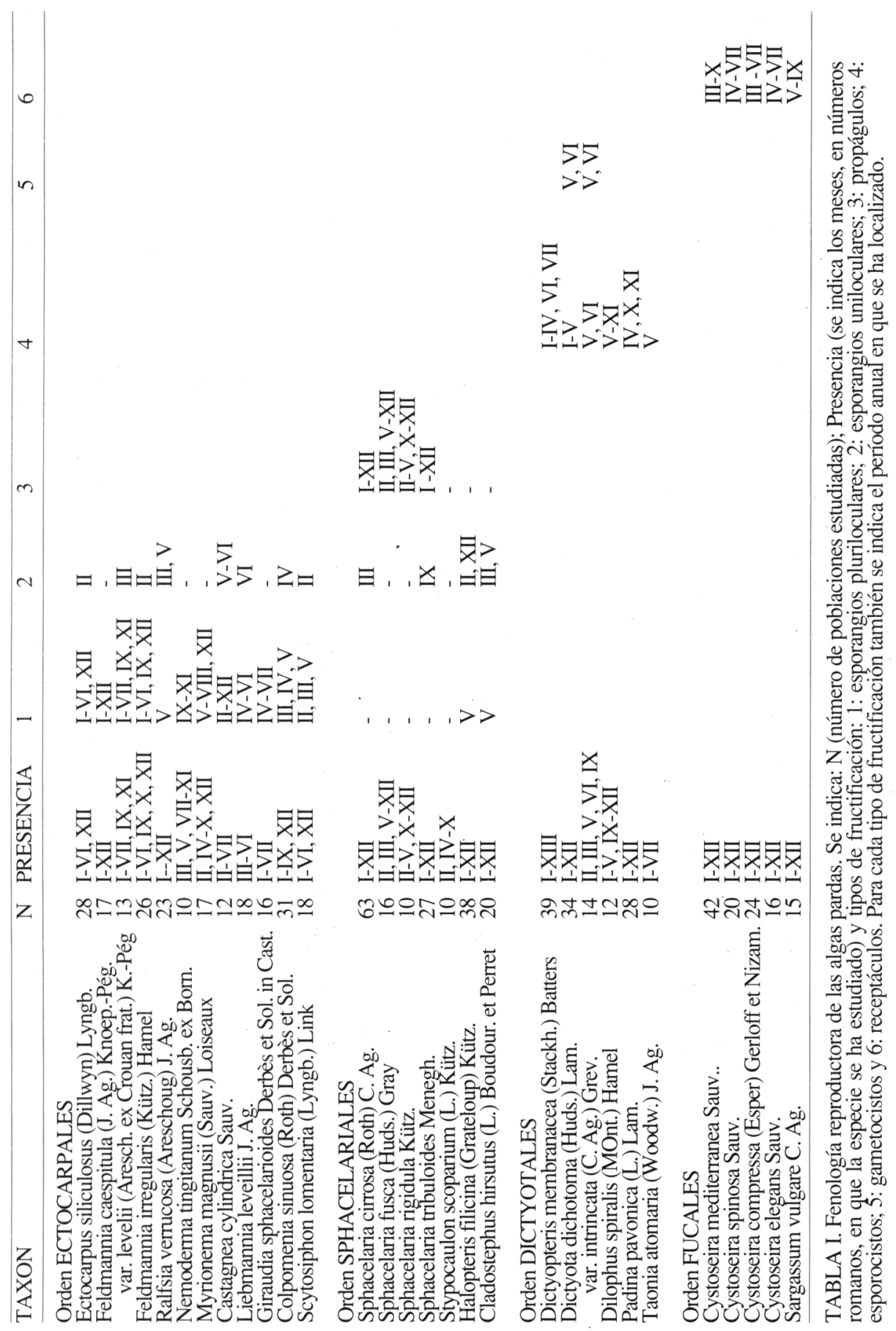




\begin{tabular}{|c|c|c|c|}
\hline TAXON & $\mathrm{N}$ & PRESENCIA & FRUCTIFICACIÓN \\
\hline $\begin{array}{l}\text { Orden TRENTEPOHLIALES } \\
\text { Pilinia rimosa Kütz. }\end{array}$ & 12 & I-VI, X-XII & - \\
\hline $\begin{array}{l}\text { Orden ULVALES } \\
\text { Enteromorpha compressa (L.) Greville } \\
\text { Enteromorpha multirramosa Bliding } \\
\text { Ulva rigida C. Ag. }\end{array}$ & $\begin{array}{l}26 \\
11 \\
16\end{array}$ & $\begin{array}{l}\text { I-XII } \\
\text { I, V-XII } \\
\text { I-XII }\end{array}$ & $\begin{array}{l}- \\
-\end{array}$ \\
\hline $\begin{array}{l}\text { Orden CTENOCLADALES } \\
\text { Phaeophila wittrockii (Wille) Nielsen } \\
\text { Phaeophila dendroides (Crouan frat.) Batters }\end{array}$ & $\begin{array}{l}14 \\
13\end{array}$ & $\begin{array}{l}\text { I-V, X-XII } \\
\text { I-XII }\end{array}$ & - \\
\hline $\begin{array}{l}\text { Orden CLADOPHORALES } \\
\text { Chaetomorpha aerea (Dillwyn) Kütz. } \\
\text { Cladophora coelothrix Kütz. } \\
\text { Cladophora dalmatica Kütz. } \\
\text { Cladophora laetevirens (Dillwyn) Kütz. } \\
\text { Cladophora lehmanniana (Lindenb.) Kütz. } \\
\text { Cladophora sericea (Huds.) Boerg. } \\
\text { Cladophoropsis modonensis (Kütz.) Boerg. } \\
\text { Anadyomene stellata (Wulfen) C. Ag. }\end{array}$ & $\begin{array}{l}18 \\
13 \\
19 \\
38 \\
16 \\
13 \\
10 \\
11\end{array}$ & $\begin{array}{l}\text { I-XII } \\
\text { I-XII } \\
\text { I-XII } \\
\text { I-XII } \\
\text { I-IV,X-XII } \\
\text { I-XII } \\
\text { VII-XII } \\
\text { I, VIII-XII }\end{array}$ & $\begin{array}{l}\text { III } \\
- \\
\text { IV, XII } \\
\text { III, IV } \\
\text { III, IV, XII } \\
\text { III, III, XII } \\
- \\
-\end{array}$ \\
\hline $\begin{array}{l}\text { Orden SIPHONOCLADALES } \\
\text { Valonia utricularis (Roth) C. Ag. }\end{array}$ & 23 & I-XII & I, IV, X \\
\hline $\begin{array}{l}\text { Orden DASYCLADALES } \\
\text { Acetabularia acetabulum (L.) Silva } \\
\text { Polyphysa parvula (Solm.) Sch. et Bula-Meller }\end{array}$ & $\begin{array}{l}11 \\
15\end{array}$ & $\begin{array}{l}\text { IV-IX } \\
\text { I, II, VII-XII }\end{array}$ & $\overline{\text { VIII, IX (cistos) }}$ \\
\hline $\begin{array}{l}\text { Orden BRYOPSIDALES } \\
\text { Derbesia tenuissima (De Not.) Crouan frat. } \\
\text { Pseudochlorodesmis fulcellata (Zanard.) Boerg. } \\
\text { Udotea petiolata (Turra) Boerg. }\end{array}$ & $\begin{array}{l}26 \\
12 \\
21\end{array}$ & $\begin{array}{l}\text { I-XII } \\
\text { I, V-XII } \\
\text { I-XII }\end{array}$ & $\begin{array}{l}\text { VII (esporangios) } \\
- \\
-\end{array}$ \\
\hline
\end{tabular}

Tabla II. Fenología reproductora y de los talos de algas verdes.

(para las feofíceas) y fructificación (de forma genérica para las clorofíceas, dado que la metodología seguida, con material formolado, no ha permitido la observación de los zooides).

Durante los meses más fríos (febrero, marzo, abril y diciembre), en los que la temperatura del agua a nivel superficial oscila entre 14 y 16 grados centígrados aparecen esporangios uniloculares en Ectocarpus siliculosus Feldmannia caespitula var. levelii, Feldmannia irregularis, Colpomenia sinuosa, Scytosiphon lomentaria, Sphacelaria cirrosa y Halopteris filicina. Presentan también esporangios uniloculares en meses más cálidos (mayo y junio con temperaturas de hasta 18 grados) Ralfsia verrucosa y Cladostephus hirsutus y solamente en los meses cálidos (mayo, junio y septiembre con temperaturas de hasta 26 grados) Castagnea cylindrica, Liebmannia leveilii y Sphacelaria tribuloides. Los esporangios pluriloculares en las especies citadas suelen tener una distribución temporal más amplia excepto en las especies del órden Sphacelariales. Es destacable en éste, la abundancia de propágulos durante todo el año. En los táxones estudiados del órden Dictyotales los esporocistos son más frecuentes que los gametocistos, excepto en Dictyota dichotoma var. intrincata, en 
que ambas estructuras reproductoras coexisten. En las fucáceas Cystoseira y Sargassum el período reproductor suele ser primaveral y estival llegando en Cystoseira mediterranea hasta los inicios del otoño (la temperatura del agua oscila entre 15 grados al comienzo del período reproductor y 26 grados al final del mismo). En las clorofíceas estudiadas las células reproductoras se observan desde febrero hasta abril y en diciembre (meses fríos) en las especies de los géneros Chaetomorpha y Cladophora. La distribución del período reproductor es más amplia y segmentaria, según los datos obtenidos, en Valonia utricularis y más canalizada hacia el verano en Polyphysa parvula y Derbesia tenuissima. En el resto de clorofíceas no se han observado fructificaciones.

\section{DISCUSIÓN}

La incidencia de esporangios uniloculares en Ectocarpales durante los meses fríos es un fenómeno general (Knoeffler-Peguy, 1977; Lee, 1980). En el Mediterráneo próximo estas estructuras reproductoras aparecen muy canalizadas en el tiempo, causa probable de que raramente aparezcan citadas. Contrariamente, los esporangios pluriloculares son citados profusamente desde las Baleares hasta el litoral de Cádiz.

En Sphacelaria, a pesar de que la formación del esporangio parece estar asociada a un fotoperíodo de días largos (Hoopen et al., 1983), tal hecho no se ve claramente verificado en el Sureste y sí en el levante español (Barceló, 1987). Los propágulos, formados con mucha mayor frecuencia que los esporangios, aparecen a temperaturas por encima de 12 grados centígrados (Lee, 1980) lo que explicaría su alta incidencia en esta región dado que las temperaturas de sus aguas no bajan nunca hasta ese valor. Por el contrario, la incidencia de propágulos es menor en litorales con aguas más frías (Málaga: Conde, 1981; Cataluña: Polo, 1978). No obstante, puede ser aventurado establecer paralelismos entre tipos de fructificación y tipos de reproducción (Cardinal, 1964; Müller, 1975; Müller \& Stache, 1989), pues zooides de esporangios uniloculares y pluriloculares pueden germinar sin evidencia de reproducción sexual.

En las fucáceas, la temperatura del mar no parece ser un factor inductor de primer orden sobre la organogénesis de estructuras reproductoras ya que éstas están presentes en un intervalo muy amplio de temperatura. Otros factores como nutrientes, ritmos fotoperiódicos o ritmos de emersión-inmersión parecen tener más importancia (Chapman \& Craige, 1977; Epiardh-Lahaye et al., 1987; Epiard-Lahaye, 1988).

En las algas verdes es de destacar la escasez de estructuras reproductoras observadas. Es atribuible al método utilizado que consistía en la observación de ejemplares formolados. A pesar de lo anterior existe una constancia en especies de la géneros Chaetomorpha y Cladophora hacia una canalización a los meses fríos de sus estructuras reproductoras, fenómeno también observado en el Mar Menor (PérezRuzafa, 1989). En Ulvales, los factores fotoperiódicos e intensidad luminosa; potenciados por la adición de nutrientes, son los inductores fundamentales en la liberación de zooides (Lersten \& Voth in Gallardo, 1984). En Derbesia tenuissima no 
han sido citados esporangios en el Mediterráneo circundante más próximo. En esta región sólo se han observado una vez durante el mes de julio, siendo la cita más próxima la del litoral gaditano (Seoane, 1965).

Entre los táxones de algas pardas y verdes estudiados fenológicamente en las áreas circundantes (Islas Baleares, litoral valenciano, malagueño y Mar Menor), en el Sureste se aprecia una más amplia distribución temporal en Ectocarpus siliculosus, Scytosiphon lomentaria (según Kristiansen \& Pedersen, 1979, es la intensidad luminosa reducida y la baja temperatura los factores que inducen la presencia de la fase tubulosa de este taxon), Colpomenia sinuosa, Sphacelaria tribuloides, Dictyota dichotoma, D. dichotoma var. intrincata (los dos últimos con igual distribución temporal que en el litoral malagueño) y Phaeophila dendroides. Presentan igual distribución temporal: Dictyopteris membranacea, Padina pavonica, Taonia atomaria, Cystoseira compressa, $C$. elegans, $C$. mediterranea (los dos últimos táxones no citados en Málaga) y Sargassum vulgare (presentando todos ellos un período reproductor más largo en el litoral balear), Chaetomorpha aerea, Udotea petiolata, Anadyomene stellata y Acetabularia acetabulum (se carece de datos de las dos últimas en el litoral malagueño.) Presenta inferior distribución temporal en relación al litoral levantino-balear Dilophus spiralis y superior a la que presenta en el litoral malagueño.

\section{BIBLIOGRAFÍA}

BARCELÓ, M.C. -1987-Estudi de les algues marines del Pais Valenciá. Tesis Doctoral. Universidad de Barcelona.

CARDINAL, A. -1964- Etude sur les Ectocarpacées de la Manche. Nova Hedwigia, 15:1-86.

CHAPMAN, A.R.O. \& J.S. CRAIGE -1977- Seasonal growth in Laminaria longicruris: relations with dissolved inorganic nutrients and internal reserves of nitrogen. Mar. Biol., 40:197-205.

CONDE, F. -1984- Catálogo de las algas macrobentónicas marinas de Málaga. Acta Bot Malacitana, 9:47-78.

EPIARD-LAHAYE, M. -1988- Effects of ammonium, nitrate and phosphate on the growth of Cystoseira stricta (Phaeophyta, Fucales) cuttings in culture. Cryptogamie. Algologie, 9(3):211-229.

EPIARD-LAHAYE, M., M. PELLEGRINI \& H. WEISS -1987- Influence des rythmes émersion-inmersion sur le développement des boutures de Cystoseira stricta Sauvageau (Phéophycées, Fucales) en Culture. Bot. Mar., 30:259-266.

GALLARDO, T. -1984- Taxonomía, ecología y fitosociología de las ulváceas del Noroeste de España. Tesis Doctoral. Universidad Complutense de Madrid.

GÓMEZ-GARRETA, A. -1983-Estudio fenológico de la vegetación marina de la Isla de Mallorca. Tesis Doctoral. Universidad Complutense de Madrid.

HENRY, E.C. -1988- Regulation of reproduction in Brown Algae by light and temperature. Bot. Mar., 31:353-357.

HOOPEN, A., S. BOS \& A.M. BREEMAN - 1983- Photoperiodic response in the formation of gametangia of the long day plant Sphacelaria rigidula (Phaeophyceae). Mar. Ecol.Progr. Ser., 13:258-289. 
KNOEPFFLER-PEGUY, M. -1977- Polymorphysme et environnement chez les Feldmannia (Ectocarpacées). Rev. Algol., N:S, 12:111-128.

KRISTIANSEN, A. \& P. PEDERSEN -1979- Studies on life history and seasonal variation of Scytosiphon lomentaria (Fucophyceae, Scytosiphonales) in Denmark. Bot.Tiddsskr., 74:3156.

LEE, R.E. - 1980- Phycology. Cambridge University Press. 458 pp.

MAGGS, C.A. \& M.D. GUIRY - 1987- Environmental control of macroalgal phenology in Plant Life in Aquatic and Amphibious Habitats. British Ecological Society, 5:359-373.

MÜLLER, D.G. -1975- Experimental evidence against sexual fusions of spores from unilocular sporangia of Ectocarpus siliculosus (Phaeophyta). Br. Phycol. J., 10:315-321.

MÜLLER, D.G. \& B. STACHE - 1989- Life History studies on Pilayella littoralis (L) Kjellman (Phaeophyceae, Ectocarpales) of diferent geographical origin. Bot. Mar., 32:71-78.

PÉREZ-RUZAFA, I.M. -1989-Fitobentos de una laguna costera. El Mar Menor. Tesis Doctoral. Universidad de Murcia.

POLO, L. -1978- Estudio sobre las algas bentónicas de la costa catalana. Tesis Doctoral. Universidad Autónoma de Barcelona.

SEOANE, J. -1965- Estudios sobre las algas bentónicas en la costa sur de la Península Ibérica (litoral de Cádiz). Inv. Pesq., 29:3-216.

SOTO, J. \& F. CONDE - 1989- Sobre la fenología reproductora de las algas rojas en el litoral del Sudeste de España (Alborán Oriental). Acta Bot. Malacitana, 14:5-12.

(Aceptado para su publicación en Junio de 1991)

Dirección del autor: Departamento de Biología Vegetal. Facultad de Biología. 30100 Murcia. 